\title{
Hydrogen Concentration Estimation Based on Density Functional Theory in Aluminum and Alpha Iron under Gaseous Hydrogen Environments
}

\author{
Y. Sakagami, R. Matsumoto, D. Alfè*, S. Taketomi**, T. Enomoto, and N. Miyazaki \\ Department of Mechanical Engineering and Science, Graduate School of Engineering, Kyoto University, Yoshida-Honmachi, \\ Sakyo-ku, Kyoto 606-8501, Japan \\ Fax: +81-75-753-5719, e-mail: matsumoto@solid.me.kyoto-u.ac.jp \\ * Department of Earth Sciences and Department of Physics and Astronomy, University College London, Gower Street, London, \\ WC1E 6BT, UK \\ ** Department of Mechanical Engineering, Graduate School of Science and Engineering, Saga University, 1 Honjo-machi, \\ Saga-city, Saga 840-8502, Japan
}

\begin{abstract}
Knowledge of the concentration of hydrogen dissolved in materials is essential for the safe design of the components of hydrogen utilization equipment and will help clarify the mechanisms of hydrogen embrittlement. Here, we present a procedure for evaluating the hydrogen concentration in a perfect lattice under practical gaseous hydrogen environments using density functional theory. We employed the condition of chemical potential equilibrium between gaseous hydrogen and dissolved hydrogen in metal as the base equation and evaluated the vibrational energy and entropy at a finite temperature using the small displacement method. The hydrogen concentrations for aluminum and alpha iron obtained by our purely computational approach agree well with experimental data.
\end{abstract}

Key words: Density Functional Theory, Solubility, Hydrogen, Lattice Vibration, Free Energy

\section{INTRODUCTION}

Hydrogen energy is expected to be used as an energy medium and source. Many facilities such as gas tanks or pipes are needed to construct a hydrogen society. However, hydrogen embrittlement, which typically reduces the toughness and ductility of metals, is considered to be a critical problem for the safe use of hydrogen. Because the degree of this effect depends on the hydrogen content of a material, it is crucial to know this content in practical environments such as high pressures ( $\sim 70 \mathrm{MPa}$ ) employed in automobile gas tanks.

The amount of hydrogen dissolved in a metal from gaseous environments is generally estimated using Sievert's law ${ }^{1}$, which states that the hydrogen concentration in metals is given by the products of solubility and the square root of the hydrogen pressure. Solubility is a function of temperature and the relation for various materials have been reported ${ }^{2-4}$. However, these experimental data can include considerable error and vary widely among reports owing to typically infinitesimal hydrogen concentration in metals and the influence of lattice defects that trap hydrogen atoms ${ }^{5,6}$. On the other hand, theoretical approaches can be an alternative way of estimating hydrogen concentrations $s^{7,8}$ because the obtained values could be separated from the effect of lattice imperfections and modified to reflect various conditions such as extremely high gaseous pressure.

In this paper, the hydrogen concentrations in $\mathrm{Al}$ and $\alpha-F e$ perfect lattices in gaseous hydrogen environments are evaluated on the basis of the condition of chemical potential equilibrium between gaseous hydrogen and dissolved hydrogen.

\section{CALCULATION THEORY}

2.1 Hydrogen concentration in the lattice

The condition of chemical potential equilibrium between gaseous hydrogen and dissolved hydrogen is expressed as ${ }^{7,8}$

$$
\frac{1}{2} \mu_{\mathrm{gas}}=\mu_{\mathrm{sol}}
$$

where $\mu_{\text {gas }}$ and $\mu_{\text {sol }}$ are the chemical potentials of a gaseous hydrogen molecule and an interstitially dissolved hydrogen atom, respectively. Here, the system consisting of a gaseous hydrogen atom and a perfect lattice is chosen as a reference state.

The value of $\mu_{\text {gas }}$ is given by the partition functions (translation $Z_{\text {trans }}$, rotation $Z_{\text {rot }}$ and vibration $Z_{\mathrm{vib}}$ ) and the ideal gas law as follows,

$$
\begin{gathered}
\mu_{\text {gas }}=-k_{\mathrm{B}} T \ln Z_{\text {trans }} Z_{\text {rot }} Z_{\text {vib }}-E_{\text {dis }} \\
Z_{\text {trans }}=\sum_{k_{x}}^{\infty} \sum_{k_{y}}^{\infty} \sum_{k_{z}}^{\infty} \exp \left\{-\frac{h^{2}}{8 M L^{2} k_{\mathrm{B}} T}\left(k_{x}^{2}+k_{y}^{2}+k_{z}^{2}\right)\right\} \\
=\left(\frac{2 \pi M k_{\mathrm{B}} T}{h^{2}}\right)^{3 / 2} \frac{k_{\mathrm{B}} T}{p} \\
Z_{\text {rot }}=\sum_{l=0,2,4, \cdots}^{\infty}(2 l+1) \exp \left\{-\frac{\Theta_{\text {rot }}}{T} l(l+1)\right\} \\
+3 \sum_{l=1,3,5, \cdots}^{\infty}(2 l+1) \exp \left\{-\frac{\Theta_{\text {rot }}}{T} l(l+1)\right\}
\end{gathered}
$$




$$
Z_{\mathrm{vib}}=\sum_{n=0}^{\infty} \exp \left\{-\frac{\Theta_{\mathrm{vib}}}{T}\left(n+\frac{1}{2}\right)\right\} .
$$

Here, $E_{\mathrm{dis}}$ is the dissociation energy of a hydrogen molecule $(4.52 \mathrm{eV})^{9}, h$ is Planck's constant, $M$ is the mass of a hydrogen molecule $\left(2.01 \times 10^{-3} \mathrm{~kg} / \mathrm{mol}\right)$, $L$ is the characteristic length, $k_{\mathrm{B}}$ is the Boltzmann constant, $T$ is the temperature, $p$ is the gas pressure, $\Theta_{\text {rot }}$ is the characteristic temperature for rotation of hydrogen molecule $(85.4 \mathrm{~K}), \Theta_{\mathrm{vib}}$ is the characteristic temperature for the vibration of hydrogen molecule $(6,100 \mathrm{~K})^{10}$.

The chemical potential of hydrogen in the dissolved state is given by Eq. (6).

$$
\mu_{\mathrm{sol}}=\Delta h_{\mathrm{sol}}-T \Delta s_{\mathrm{sol}}
$$

Here, $\Delta h_{\text {sol }}$ and $\Delta s_{\text {sol }}$ are changes in the enthalpy and entropy of the lattice, respectively, due to dissolved hydrogen.

Change in enthalpy, $\Delta h_{\text {sol }}$, can be expressed as follows,

$$
\Delta h_{\mathrm{sol}}=\Delta E_{\mathrm{lat}}+\Delta E_{\mathrm{vib}},
$$

where $\Delta E_{\text {lat }}$ is change in the ground state energy $\left(\Delta E_{\text {lat }}=-1.60 \mathrm{eV}\right.$ for $\mathrm{Al}^{9}$ and $\Delta E_{\text {lat }}=-2.06 \mathrm{eV}$ for $\left.\alpha-\mathrm{Fe}^{11}\right)$ and $\Delta E_{\mathrm{vib}}$ is change in the vibrational energy. The effect of the volume expansion due to the dissolved hydrogen is neglected here because the influence is sufficiently small.

The change in entropy $\Delta s_{\text {sol }}$ in Eq. (6) can be represented as follows.

$$
\Delta s_{\mathrm{sol}}=s_{\mathrm{con}}+\Delta s_{\mathrm{vib}}
$$

Here, $s_{\text {con }}$ is the configurational entropy of hydrogen atoms in the lattice and $\Delta s_{\text {vib }}$ is change in the vibrational entropy due to dissolved hydrogen. The configurational entropy is expressed as

$$
s_{\text {con }} \approx-k_{\mathrm{B}} \ln \frac{x}{r-x},
$$

where $r$ is the number of interstitial sites per lattice atom $[r=2$ for the tetrahedral site in the fcc lattice (hydrogen atoms in $\mathrm{Al}$ ) and $r=6$ for the tetrahedral site in the bcc lattice (hydrogen atoms in $\alpha-\mathrm{Fe}$ )] and $x$ is the concentration of hydrogen (atomic ratio of hydrogen atoms to lattice atoms). Here, we assumed that the concentration of dissolved hydrogen is sufficiently low, and hence, we can neglect hydrogen-hydrogen interactions. Using Eqs. (7)-(9), Eq. (6) is rewritten as

$$
\mu_{\mathrm{sol}}=\Delta E_{\mathrm{lat}}+\Delta E_{\mathrm{vib}}+k_{\mathrm{B}} T \ln \frac{x}{r-x}-T \Delta s_{\mathrm{vib}} .
$$

By substituting Eqs. (2) and (10) in Eq. (1), the hydrogen concentration $x$ is obtained as follows.

$$
\begin{aligned}
\frac{x}{r-x}= & \exp \left(\frac{\Delta s_{\text {vib }}}{k}\right) \\
& \times \exp \left(-\frac{\Delta E_{\text {lat }}+\Delta E_{\text {vib }}+1 / 2 E_{\text {dis }}}{k T}\right) \frac{1}{\sqrt{Z_{\text {trans }} Z_{\text {rot }} Z_{\text {vib }}}}
\end{aligned}
$$

The expression of the two unknown terms, i.e., change in vibrational energy $\Delta E_{\mathrm{vib}}$ and change in entropy $\Delta s_{\mathrm{vib}}$, are presented in the next section.

2.2 Evaluation of the vibrational properties of the lattice

For an atomic system that can be represented by repeating a unit cell consisting of $n$ atoms, each atom can be differentiated by the two indices of cell $i$ and atom $\mu^{12}$. If the system is near its minimum energy configuration, the change in potential with small atomic displacements is expressed using only a quadratic term, as shown in Eq. (12).

$$
E=\frac{1}{2} \sum_{i, j, \mu, \nu} \boldsymbol{u}_{j \nu}^{\mathrm{T}} \Phi_{i \mu, j v} \boldsymbol{u}_{i \mu}
$$

Here, $\boldsymbol{u}_{i \mu}$ denotes the displacement of atom $\mu$ in cell $i$ from the equilibrium position and $\boldsymbol{\Phi}_{i \mu j v}$ is the force-constant matrix, which is given by the second derivative of the potential energy. The force acting on an atom $\boldsymbol{F}_{i \mu}$ is expressed as follows.

$$
\boldsymbol{F}_{i \mu}=-\frac{\partial E}{\partial \boldsymbol{u}_{i \mu}}=-\sum_{j, v} \Phi_{i \mu, j v} \boldsymbol{u}_{j v}
$$

Therefore, the equation of motion with respect to atom $\mu$ in cell $i$ is given by Eq. (14) and the general solution of displacement is expressed by Eq. (15).

$$
\begin{gathered}
m_{\mu} \ddot{\boldsymbol{u}}_{i \mu}=\boldsymbol{F}_{i \mu}=-\sum_{j, v} \Phi_{i \mu, j v} \boldsymbol{u}_{j v} \\
\boldsymbol{u}_{i \mu}=\frac{1}{\sqrt{m_{\mu}}} \boldsymbol{u}_{0 \mu} \exp \left(i \boldsymbol{k} \cdot \boldsymbol{R}_{i \mu}-i \omega_{\boldsymbol{k}} t\right)
\end{gathered}
$$

Here, $m_{\mu}$ is the mass of atom $\mu, \boldsymbol{u}_{0 \mu}$ is the displacement vector, $\boldsymbol{k}$ is the wave vector, $\boldsymbol{R}_{i \mu}$ is the equilibrium position of atom $\mu$ in cell $i$, and $\omega_{\boldsymbol{k}}$ is the frequency. We can obtain $\boldsymbol{u}_{0 \mu}$ and $\omega_{k}$ as the eigenvector and the square root of the eigenvalue of the dynamical matrix, respectively, given by

$$
\boldsymbol{D}_{\mu, v}=\frac{1}{\sqrt{m_{\mu} m_{\nu}}} \sum_{j} \Phi_{i \mu, j v} \exp \left\{i \boldsymbol{k} \cdot\left(\boldsymbol{R}_{j v}-\boldsymbol{R}_{i \mu}\right)\right\} .
$$

The dimensions of the dynamical matrix are $3 n \times 3 n$, and hence, the vibrational mode has $3 n$ solutions, which are differentiated by index, $j$, as $\omega_{\boldsymbol{k}, j}$.

The vibrational energy $E_{\boldsymbol{k}, j}$ and entropy $s_{\boldsymbol{k}, j}$ for frequency $\omega_{k, j}$ are derived using Eqs. (17) and (18).

$$
E_{k, j}=\frac{\hbar \omega_{k, j}}{2} \operatorname{coth}\left(-\frac{\hbar \omega_{k, j}}{k_{\mathrm{B}} T}\right)
$$


$S_{k, j}=k_{\mathrm{B}} \frac{\hbar \omega_{k, j}}{k_{\mathrm{B}} T} \frac{\exp \left(-\frac{\hbar \omega_{k, j}}{k_{\mathrm{B}} T}\right)}{1-\exp \left(-\frac{\hbar \omega_{k, j}}{k_{\mathrm{B}} T}\right)}-k_{\mathrm{B}} \ln \left(1-\exp \left(-\frac{\hbar \omega_{k, j}}{k_{\mathrm{B}} T}\right)\right)$

The vibrational energy $E_{\text {vib }}$ and entropy $s_{\text {vib }}$ per primitive cell are obtained by summing $E_{k, j}$ and $s_{k, j}$, respectively, over all the vibration modes $j$ and integrating over the wave vector $\boldsymbol{k}$ in the Brillouin zone. Finally, $\Delta E_{\mathrm{vib}}$ and $\Delta s_{\text {vib }}$ are obtained as the difference between $E_{\text {vib }}$ and $s_{\text {vib }}$ before and after hydrogen is introduced into the lattice.

\subsection{Calculation method}

We used the small displacement method to construct the dynamical matrix and density functional theory (DFT) to calculate the force acting on each atom.

The DFT calculations were performed using the Vienna $a b$ initio simulation package ${ }^{13-15}$ in the spin-polarized generalized gradient approximation ${ }^{16}$ for electron exchange and correlation. Ion-electron interactions are described by the ultrasoft pseudopotential method ${ }^{17}$ for the $\mathrm{Al}-\mathrm{H}$ system and by Blöchl's projector augmented wave method ${ }^{18}$, which is an all-electron method in the frozen-core approximation, for the Fe-H system. The Monkhorst-Pack scheme ${ }^{19}$ is used to define the $k$-points and the conjugate-gradient method is employed for the relaxation of the initial structure. The $k$-point mesh is $18 \times 18 \times 18$ for a primitive cell of $\mathrm{Al}$ and $\alpha-\mathrm{Fe}$. The cutoff energy is 600 $\mathrm{eV}$ for the $\mathrm{Al}-\mathrm{H}$ system and $425 \mathrm{eV}$ for the $\mathrm{Fe}-\mathrm{H}$ system.

The calculations of the vibrational mode and vibrational properties are performed using $\mathrm{PHON}^{12}$ with the central difference scheme. To avoid numerical errors, models of the same size (e.g., $\mathrm{Al}_{n}$ and $\mathrm{Al}_{n} \mathrm{H}$ ) are used to calculate $\Delta E_{\mathrm{vib}}$ and $\Delta s_{\mathrm{vib}}$.

\section{HYDROGEN CONCENTRATION IN ALUMINUM}

The density of phonon states in a pure Al lattice and in a lattice containing a hydrogen atom are shown in Fig.1. They are calculated using models of $\mathrm{Al}_{27}$ consisting of $3 \times 3 \times 3$ primitive cells and of $\mathrm{Al}_{27} \mathrm{H}$ containing one interstitial hydrogen. The density of the states of pure $\mathrm{Al}$ shows two peaks around $5 \mathrm{THz}$ and $8 \mathrm{THz}$ and all values are distributed below $10 \mathrm{THz}$. On the other hand, in the density of states of $\mathrm{Al}_{27} \mathrm{H}$, two peaks appear around $25 \mathrm{THz}$ and $10 \mathrm{THz}$, and the two peaks for pure $\mathrm{Al}$ are lower. These differences are considered to result from the presence of dissolved hydrogen. The displacement vectors of the two high-frequency modes at $\Gamma$ point are shown in Fig.2. Here, the results for $\mathrm{Al}_{8} \mathrm{H}$ are shown for viewability. The highest frequency peak $(\approx 25 \mathrm{THz})$ and the second peak $(\approx 10 \mathrm{THz})$ arise from hydrogen dominant vibration and vibration of $\mathrm{Al}$ atoms around the dissolved hydrogen, respectively.

Changes in the vibrational energy $\Delta E_{\mathrm{vib}}$ and entropy $\Delta s_{\text {vib }}$ with the introduction of hydrogen are calculated with models of up to $6 \times 6 \times 6$ supercells and the results are shown in Figs. 3 and 4, respectively. Fig.5 shows the hydrogen concentrations calculated using Eq. (11) under $p=70 \mathrm{MPa}$. The hydrogen concentrations obtained by Ichimura et al. $^{2}$ are also shown in the approximate line expressed by Eq. (19) with $\alpha_{\mathrm{Al} \_ \text {exp }}=1.25 \times 10^{-5} \mathrm{~Pa}^{-1 / 2}$ and $\beta_{\mathrm{Al} \_ \text {exp }}=7,690 \mathrm{~K}$. The function was extrapolated from the measured data obtained above $700 \mathrm{~K}$.

$$
x=\alpha_{\mathrm{Al} \_ \text {exp }} \exp \left(-\beta_{\mathrm{Al} \_ \text {exp }} / T\right) \times \sqrt{p}
$$

The experimental data includes the influence of hydrogen trapped at lattice defects. However, we confirmed that this effect on the absolute amount of hydrogen is very small for $\mathrm{Al}^{9}$ and $\alpha-\mathrm{Fe}^{20}$.

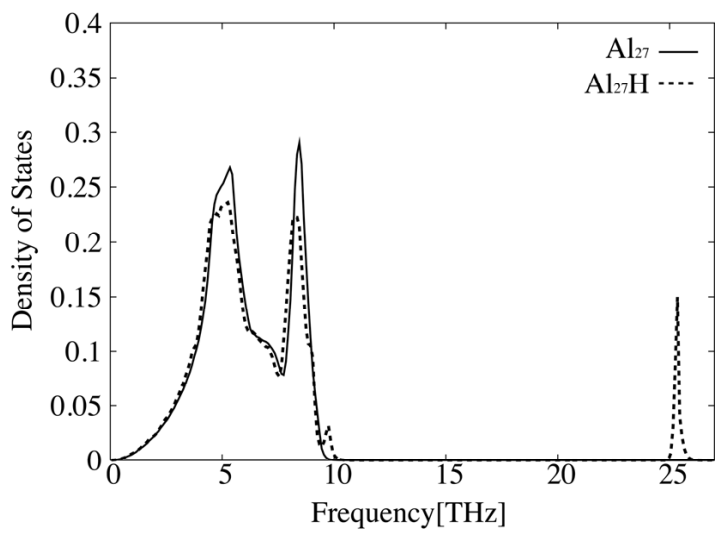

Fig.1. Density of phonon states in pure $\mathrm{Al}$ lattice $\left(\mathrm{Al}_{27}\right)$ and $\mathrm{Al}$ lattice containing one hydrogen $\left(\mathrm{Al}_{27} \mathrm{H}\right)$.

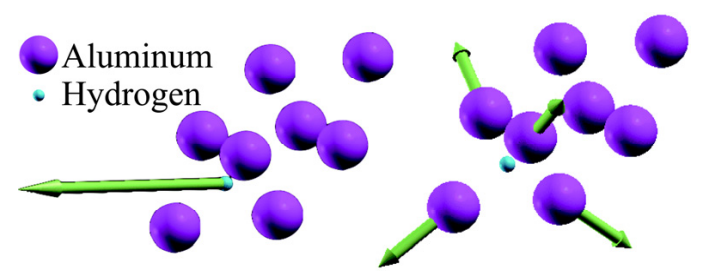

Fig.2. High-frequency vibrational modes in $\mathrm{Al}_{8} \mathrm{H}$ model (left: $25 \mathrm{THz}$ mode, right: $10 \mathrm{THz}$ mode).

The vibrational energy curves in Fig. 3 converge well with the model size, but the vibrational entropy curves shown in Fig.4 do not converge. This is because the vibrational entropy is a more sensitive function of frequency than the vibrational energy, especially in low-frequency modes. Hence, the obtained vibrational entropy includes errors of some tens of percent. However, we can confirm the same tendency in the temperature dependence for each model size, and thus, as shown in Fig.5, the gradients of the calculated hydrogen concentrations are very close to each other. In addition, although the vibrational entropy is slightly affected by model size, the calculated hydrogen concentrations agree well with the experimental data. We can conclude that the dissolved hydrogen in $\mathrm{Al}$ lattice from pure gaseous hydrogen environments is infinitesimal.

To evaluate the influence of the vibrational energy and entropy of lattice, the hydrogen concentrations, when their values are neglected $\left(\Delta E_{\text {vib }}\right.$ and/or $\Delta s_{\text {vib }}$ are equal to 0), are shown in Fig.6. Here, the vibrational energy and entropy of hydrogen molecule are considered, 
because they are included in the partition functions in Eq (2). Ignoring the change in vibrational energy of lattice increases the hydrogen concentration by about two orders of magnitude and the vibrational entropy decreases by about one order of magnitude. The calculated hydrogen concentrations agree with the experimental values only when both the changes are considered. And thus, although calculations of vibrational properties require computational power, they are essential for the accurate estimation of the hydrogen concentration. In Fig.5, the calculated hydrogen concentrations have a weak curvature, but under the approximation with the function in Eq. (19) for the curve of $\mathrm{Al}_{125} \mathrm{H}$ model, we can obtain $\alpha_{\mathrm{Al} \text { _cal }}=6.18 \times 10^{-6}$ $\mathrm{Pa}^{-1 / 2}$ and $\beta_{\mathrm{Al} \text { cal }}=7,623 \mathrm{~K}$ for the approximate line.

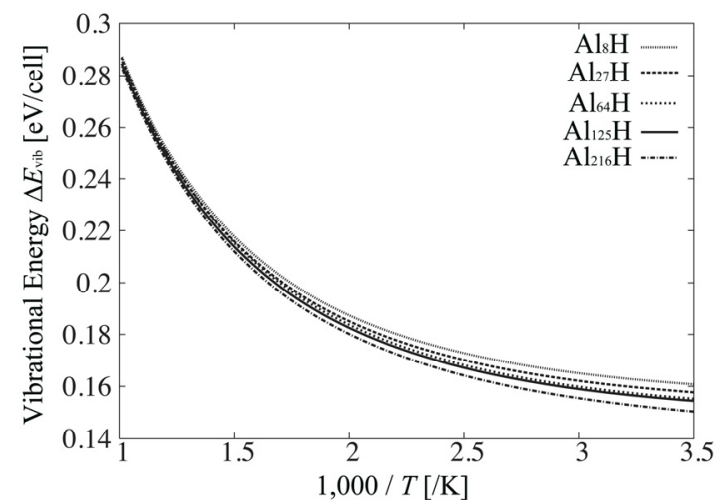

Fig.3. Change in vibrational energy $\Delta E_{\text {vib }}$ of Al due to dissolved hydrogen.

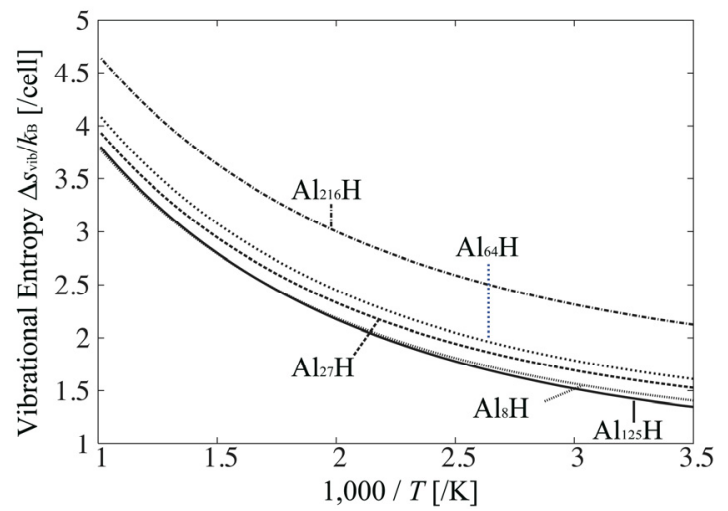

Fig.4. Change in vibrational entropy $\Delta s_{\text {vib }}$ of $\mathrm{Al}$ due to dissolved hydrogen.

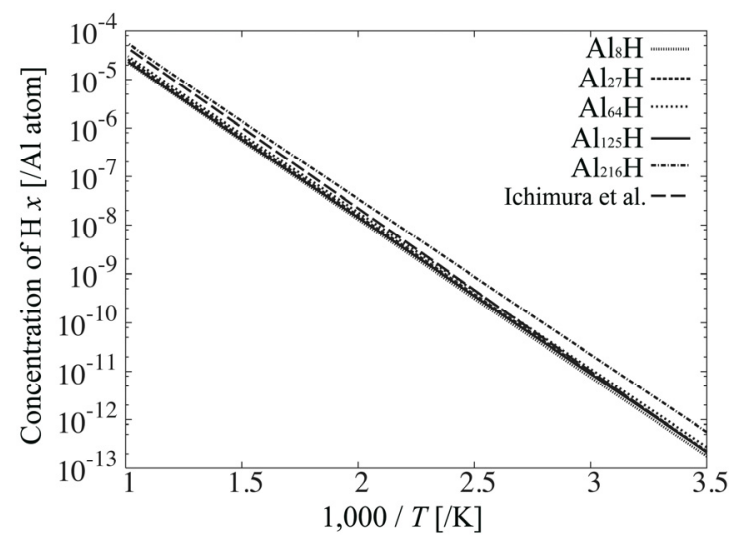

Fig.5. Hydrogen concentration $x$ in $\mathrm{Al}$ at $p=70 \mathrm{MPa}$.

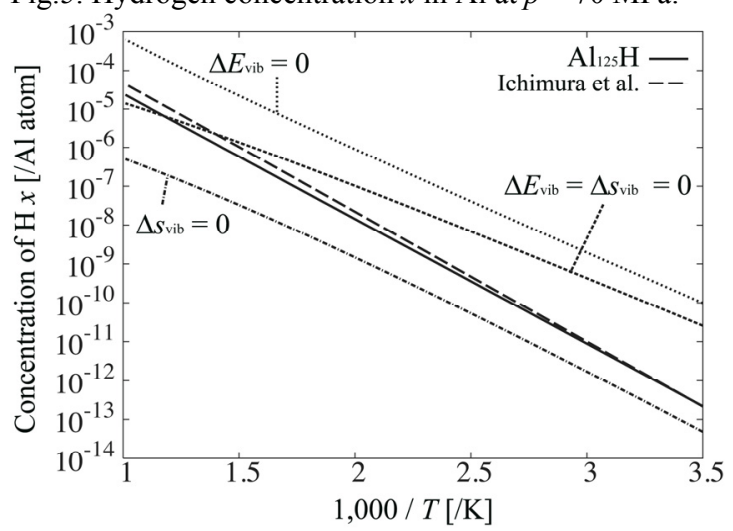

Fig.6. Influence of vibrational energy $\Delta E_{\mathrm{vib}}$ and entropy $\Delta s_{\text {vib }}$ on hydrogen concentration in Al.

\section{HYDROGEN CONCENTRATION IN ALPHA IRON}

The density of phonon states in a pure $\alpha-\mathrm{Fe}$ lattice $\left(\mathrm{Fe}_{64}\right)$ and in a lattice containing a hydrogen atom $\left(\mathrm{Fe}_{64} \mathrm{H}\right)$ are shown in Fig.7. Three peaks appear around 10, 29, and $43 \mathrm{THz}$ with the introduction of hydrogen. The $\mathrm{Fe}$ atoms around the dissolved hydrogen vibrate mainly in the $10 \mathrm{THz}$ mode, and the dissolved hydrogen vibrates mainly in the 29 and $43 \mathrm{THz}$ modes. The branches of the high-frequency modes occur because the tetrahedral site in the bcc lattice is asymmetrical.

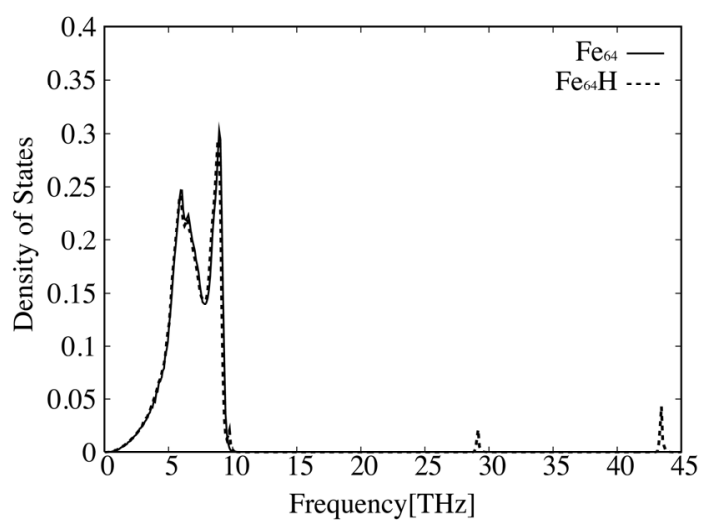

Fig.7. Density of phonon states in pure $\alpha-\mathrm{Fe}$ lattice $\left(\mathrm{Fe}_{64}\right)$ and $\alpha-\mathrm{Fe}$ lattice containing a hydrogen $\left(\mathrm{Fe}_{64} \mathrm{H}\right)$.

Changes in vibrational energy and entropy up to the 4 $\times 4 \times 4$ supercell model (Fe: 64 atoms) are shown in Figs. 8 and 9, respectively. Fig.10 shows the calculated hydrogen concentratin under $p=70 \mathrm{MPa}$. In Fig.10, the experimental data, which were extrapolated from measured data obtained above $300 \mathrm{~K}$, are also shown with error range $\mathrm{e}^{3}$. The approximation line is expressed using the function in Eq. (19) by replacing $\alpha_{\mathrm{Al} \text { exp }}$ and $\beta_{\mathrm{Al} \_ \text {exp }}$ with $\alpha_{\mathrm{Fe} \_ \text {exp }}$ and $\beta_{\mathrm{Fe} \_ \text {exp }}$, respectively. 


$$
\begin{aligned}
& \alpha_{\mathrm{Fe} \_ \text {exp }}=1.85\left(\begin{array}{l}
+10.3 \\
-1.57
\end{array}\right)[1 / \sqrt{\mathrm{Pa}}] \\
& \beta_{\text {Fe_exp }}=3440 \pm 341[\mathrm{~K}]
\end{aligned}
$$

The calculated concentrations of each model were included in the error span of the experimental data. We can confirm that the dissolved hydrogen in $\alpha-\mathrm{Fe}$ from pure hydrogen gaseous environments is much higher than that in $\mathrm{Al}$ especially at lower temperature.

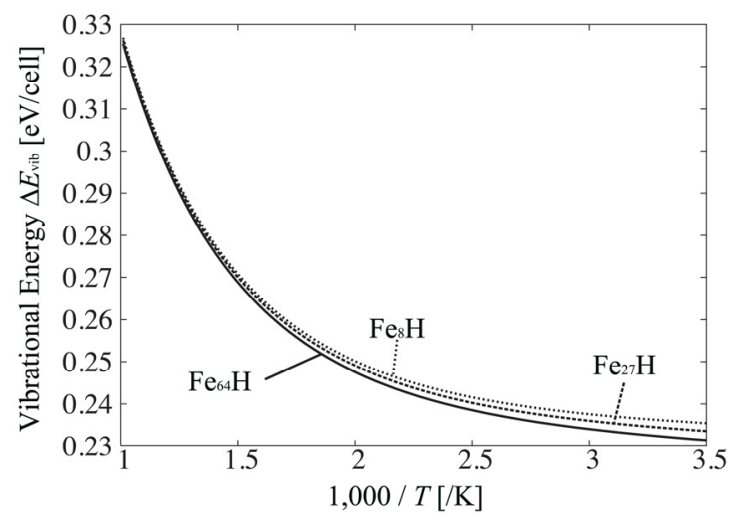

Fig.8. Change in vibrational energy $\Delta E_{\text {vib }}$ in $\alpha-\mathrm{Fe}$ due to dissolved hydrogen.

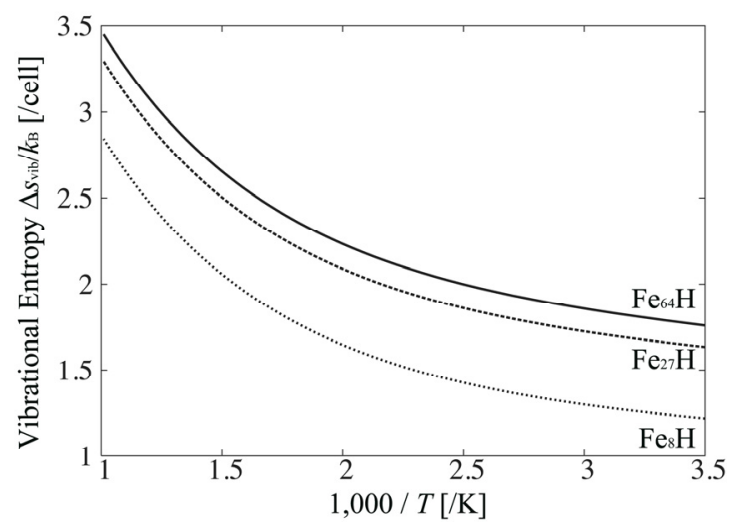

Fig.9. Change in vibrational entropy $\Delta s_{\text {vib }}$ in $\alpha-F e$ due to dissolved hydrogen.

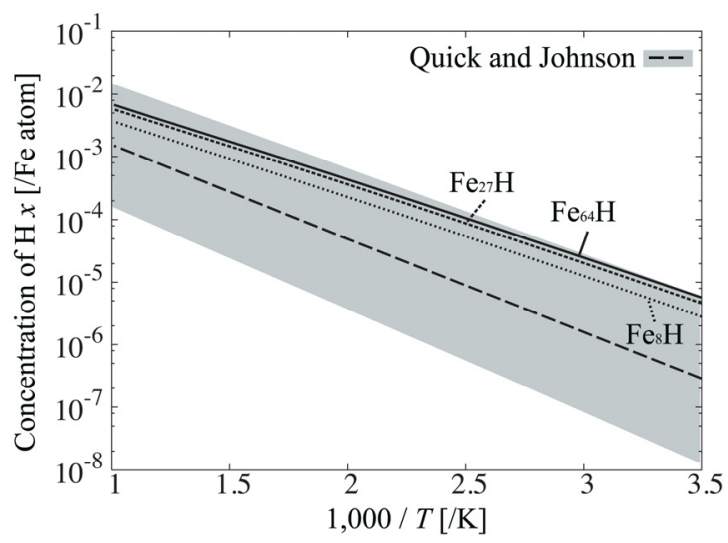

Fig.10. Hydrogen concentration $x$ in $\alpha-\mathrm{Fe}$ at $p=70$ MPa.

The hydrogen concentrations when vibrational energy and/or entropy of lattice are neglected are shown in Fig.11. The gradients of the calculated hydrogen concentrations for $\alpha-\mathrm{Fe}$ tend to be more markedly affected by the vibrational energy than those for the $\mathrm{Al}-\mathrm{H}$ system. This is because $\mathrm{Fe}$ atoms affect the motion of the dissolved hydrogen atom more strongly as compared with $\mathrm{Al}$ atoms since much higher frequency modes appear in Fig.7 (compared with Fig.1). Again, the calculated hydrogen concentrations represent the experimental data most accurately when changes in both the vibrational energy and entropy are considered. The concentration of hydrogen $x$ calculated using a model of $\mathrm{Fe}_{64} \mathrm{H}$ can be approximated by the function of Eq. (19) with $\alpha_{\mathrm{Fe} \text { cal }}=9.01 \mathrm{~Pa}^{-1 / 2}$ and $\beta_{\mathrm{Fe} \_ \text {cal }}=2,773 \mathrm{~K}$.

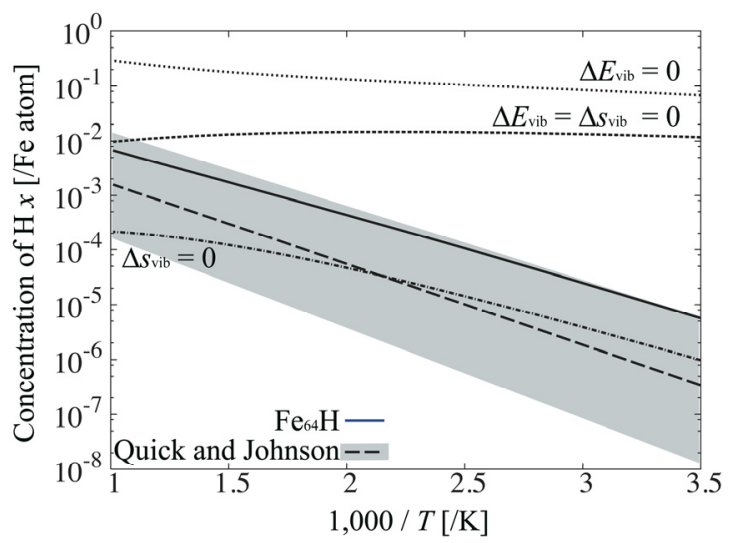

Fig.11. Influence of vibrational energy $\Delta E_{\text {vib }}$ and entropy $\Delta s_{\mathrm{vib}}$ on hydrogen concentration in $\alpha-\mathrm{Fe}$.

\section{CONCLUSIONS}

We presented a procedure for evaluating the hydrogen concentration in metals under gaseous hydrogen environments by a purely computational approach. The calculated hydrogen concentrations in $\mathrm{Al}$ and $\alpha-\mathrm{Fe}$ showed good quantitative agreement with experimental data only when change in both vibrational energy and entropy of lattice were considered. Although the calculations of vibrational properties require computational power, they can be performed by monotonous repetitive tasks.

Using the lattice hydrogen concentration obtained here and the interaction energy between hydrogen atoms and lattice defects, we can approximately evaluate the hydrogen concentration around lattice defects ${ }^{9}, 21-23$. Moreover, the calculation method presented in this paper can be applied to various solute and solvent systems.

\section{ACKNOWLEDGMENTS}

This work was supported by the JSPS Institutional Program for Young Researcher Overseas Visits.

\section{REFERENCES}

[1] A. Sieverts and W. Krumbhaar, Ber. Dtsch. Chem. Ges., 40, 893-900 (1910).

[2] M. Ichimura, H. Katsuta, Y. Sasajima and M. Imabayashi, J. Phys. Chem. Solids., 49, 1259-67 (1988). [3] N. R. Quick and H. H. Johnson, Acta Metall., 26, 903-7 (1978).

[4] S. A. Steward, "Review of Hydrogen Isotope 
Permeability Through Materials", Lawrence Livermore National Laboratory Report, Livermore (1983) pp. 1-12. [5] J. R. G. D. Silva and R. B. Mclellan, J. Less-Common Met., 50, 1-5 (1976).

[6] N. Z. Carr and R. B. McLellan, J. Phys. Chem. Solids., 67, 1797-802 (2006).

[7] H. Sugimoto and Y. Fukai, Acta Metall. Mater., 40, 2327-36 (1992)

[8] H. Ogawa, J. Phys. Chem. C, 114, 2134-43, (2010).

[9] T. Enomoto, R. Matsumoto, S. Taketomi and N. Miyazaki, J. Soc. Mater. Sci., Japan, 59, 596-603 (2010).

[10] K. Ikeda, "Statistical Thermodynamics", Kyoritsu Shuppan, Tokyo (1975) pp. 254-61.

[11] D. E. Jiang and E. A. Carter, Phys. Rev. B, 70, 064102, (2004).

[12] D. Alfè, Comp. Phys. Commun., 180, 2622-33 (2009).

[13] G. Kresse and J. Hafner, Phys. Rev. B, 48, 13115-18 (1993).

[14] G. Kresse and J. Furthmüller, Phys. Rev. B, 54, 11169-86 (1996).

[15] G. Kresse and J. Furthmüller, Comput. Mater. Sci., 6, 15-50 (1996).

[16] J. P. Perdew and Y. Wang, Phys. Rev. B, 45, 13244-49 (1992).

[17] D. Vanderbilt, Phys. Rev. B, 41, 7892-95 (1990).

[18] P. E. Blöchl, Phys. Rev. B, 50, 17953-79 (1994).

[19] H. J. Monkhorst and J. D. Pack, Phys. Rev. B, 13, 5188-92 (1976).

[20] H. Kotake, R. Matsumoto, S. Taketomi and N. Miyazaki, Int. J. Pres. Ves. Pip., 85, 540-9 (2008)

[21] D. McLean, "Grain boundaries in metals", Clarendon Press, Oxford (1957).

[22] R. A. Oriani, Acta Metall., 18, 147-57 (1970).

[23] S. Taketomi, R. Matsumoto and N. Miyazaki, Acta Mater., 56, 3761-69 (2008).

(Received March 22, 2011; Accepted April 22, 2011) 